Revue des patrimoines

$31 \mid 2017$

Patrimoines de la santé : essais de définition - enjeux de conservation

\title{
Aperçu sanitaire et architectural des bâtiments de transit pour les émigrants européens
}

A sanitary and architectural survey of transit buildings for European emigrants

\section{Anne Bosser}

\section{(2) OpenEdition}

\section{Journals}

Édition électronique

URL : http://journals.openedition.org/insitu/14030

DOI : $10.4000 /$ insitu. 14030

ISSN : $1630-7305$

Éditeur

Ministère de la Culture

Référence électronique

Anne Bosser, « Aperçu sanitaire et architectural des bâtiments de transit pour les émigrants

européens », In Situ [En ligne], 31 | 2017, mis en ligne le 21 février 2017, consulté le 10 octobre 2020.

URL : http://journals.openedition.org/insitu/14030; DOI : https://doi.org/10.4000/insitu.14030

Ce document a été généré automatiquement le 10 octobre 2020.

\section{cc) () $९$}

In Situ Revues des patrimoines est mis à disposition selon les termes de la licence Creative Commons Attribution - Pas d'Utilisation Commerciale - Pas de Modification 4.0 International. 


\section{Aperçu sanitaire et architectural des bâtiments de transit pour les émigrants européens}

A sanitary and architectural survey of transit buildings for European emigrants

Anne Bosser

Du milieu du XIX ${ }^{e}$ siècle au déclenchement de la Première Guerre mondiale, l'épisode de "la grande émigration européenne» donne lieu à un exode massif de migrants déshérités, en partance pour les Amériques ${ }^{1}$. Cet exode migratoire, estimé à plus de 50 millions d'individus entre 1850 et $1930^{2}$, est orchestré par les compagnies de navigation naissantes qui, accréditées et subventionnées par les gouvernements de leurs pays respectifs, prennent conscience de l'intérêt commercial complémentaire à un simple transport maritime. Elles revendiquent le transport de l'émigrant indigent en s'octroyant l'exclusivité de la réglementation de l'émigration dans les principaux ports de départ européens. Or, si cette activité lucrative du phénomène migratoire est tout à fait avérée, il est moins facile d'envisager que les compagnies maritimes internationales aient également fourni une double réponse sanitaire et architecturale dès 1891 (fig. 1). 
Figure 1

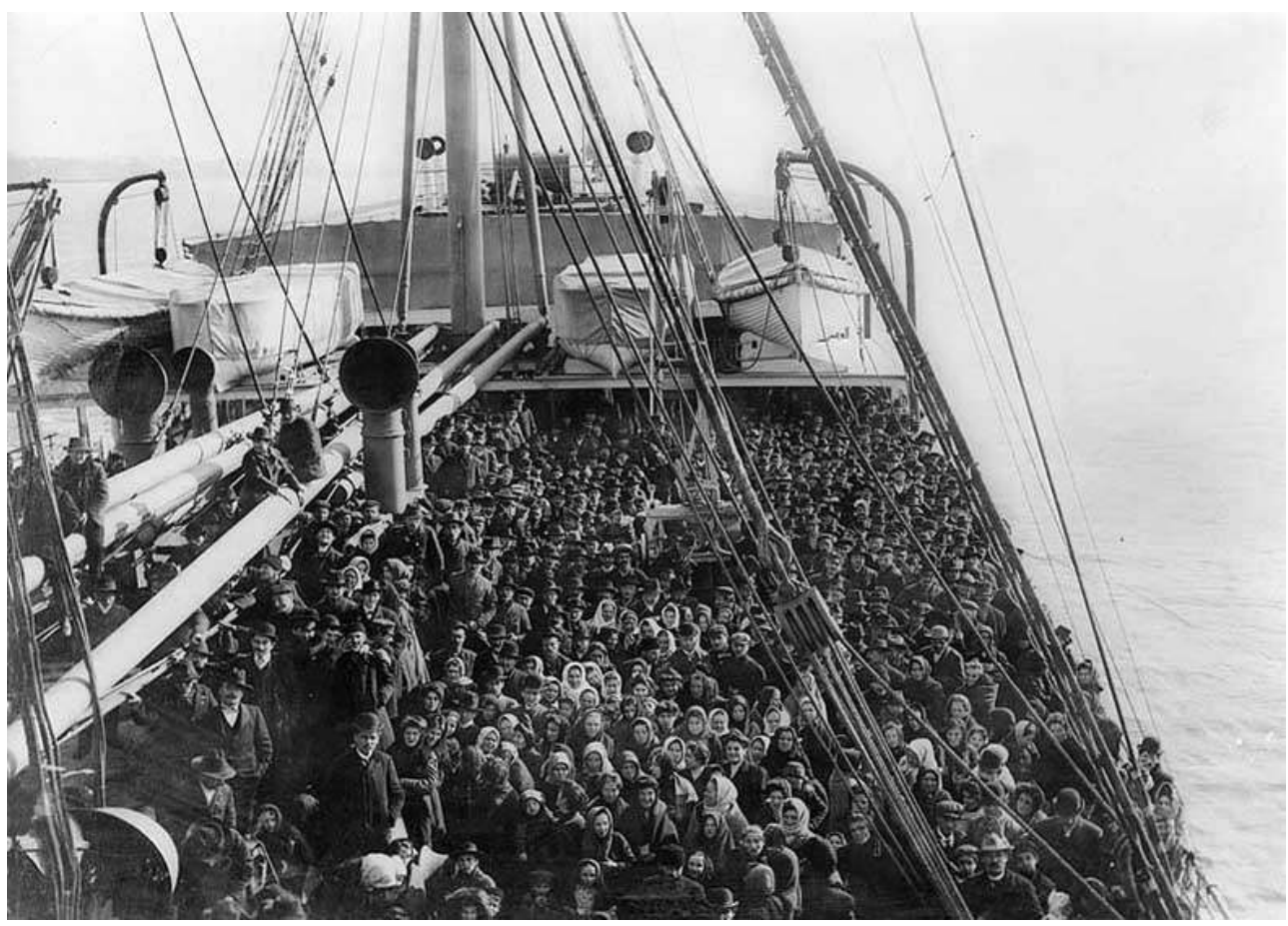

Émigrants sur un bateau transatlantique.

(C) THE STATUE OF LIBERTY-ELLIS ISLAND FOUNDATION, INC./NATIONAL PARK SERVICE.

2 En effet, cette année marque un tournant important pour les sociétés maritimes transatlantiques puisqu'elle correspond à une véritable prise de conscience sanitaire de part et d'autre de l'Atlantique. Cela se manifeste, sur le territoire américain, par l'ouverture de la station sanitaire d'Ellis Island à New York et, en Europe, par celle de Ruhleben, près de Berlin. Parallèlement, le développement d'un système de logistique, situé sur le corridor de transit à travers l'Allemagne, est mis en place aux postes sanitaires installés soit aux frontières russes (Bajoren, Illowo, Insterburg, Posen, Tilsit ${ }^{3}$ ) soit aux frontières autrichiennes (Bingerbrück, Myslowitz, Ratibor ${ }^{4}$ ). Controlées par la police et le gouvernement fédéral allemand, ces étapes, incontournables et intermédiaires vers les ports de Hambourg, de Brême et de Rotterdam ainsi que vers d'autres ports d'Europe occidentale, deviennent des lieux d'hébergement et de désinfection. Ils interceptent, durant une semaine de "quarantaine ", des milliers de Juifs, Polonais, Ukrainiens et autres migrants des empires russe et austro-hongrois pour leur faire subir les redoutables examens sanitaires en vigueur. Le témoignage de la jeune Mary Antin et de sa famille est très révélateur des conditions dans lesquelles ces examens se déroulent. Elle décrit en ces termes une vraie scène de chaos :

«Des Allemands, certains en blouse blanche, précipitent les migrants hors d'un train, les hommes séparés des femmes et des enfants, et jettent les bagages sur un gros tas [...]. Les migrants désorientés et terrifiés sont conduits dans un petit immeuble. Ils sont forcés de se déshabiller et de subir la désinfection dans une douche primitive $»^{5}$.

3 Ces inspections sont non seulement maintenues par les deux compagnies maritimes hanséatiques (Hamburg Amerika Linie et Norddeutscher Lloyd) mais également par les compagnies transatlantiques concurrentes, qui achètent un droit de passage au gouvernement allemand pour la libre circulation de ses émigrants via les frontières et 
le territoire germaniques. Toutefois, d'après les statistiques de la Hamburg Amerika Linie, quelques refus sont comptabilisés ${ }^{6}$ aux postes de contrôle germaniques : en 1907, on dénombre 664 émigrants rejetés aux postes frontières. Ces évictions sont dues, dans $74,3 \%$ des cas, à des raisons médicales dont les deux principales sont soit une maladie des yeux (le trachome, maladie oculaire contagieuse pouvant conduire à la cécité), soit des lésions inflammatoires (granulomes). Nous pouvons également noter que $16,2 \%$ des émigrants sont refusés pour des motifs d'apparence physique ou psychique, arguments qui pourraient être repris dès l'entrée sur le territoire des États-Unis et qui justifieraient un renvoi immédiat.

4 À partir de la fin du XIX ${ }^{\mathrm{e}}$ siècle, l'organisation de ce contrôle intermédiaire des deux côtés de l'Atlantique, de Ruhleben à Ellis Island, donne une impulsion décisive aux plus importantes compagnies maritimes européennes. Elles décident de s'engager en Europe dans une politique conjointe, caractérisée par la construction de dispositifs d'accueil et d'hébergement à vocation sanitaire et sociale, le but étant d'accueillir, sélectionner, désinfecter, contrôler puis exporter cet abondant fret humain avant la traversée transatlantique. Pour mettre en œuvre cette prophylaxie, elles missionnent des architectes investis dans le domaine de la salubrité. Ces nouveaux maîtres d'œuvre, soucieux de se conformer aux prérogatives hygiénistes, élaborent de nouveaux programmes constructifs dans lesquels la notion de désinfection occupe une place importante. Cela se traduit par des recommandations spécifiques qui concernent la totalité des édifices, de leur implantation urbaine au choix des matériaux de construction jusqu'aux détails du second œuvre dans l'organisation des espaces intérieurs.

5 Du point de vue urbanistique, on choisit soigneusement l'emplacement de la construction de ces lieux de transit dédiés aux émigrants. Ils doivent être situés en dehors du centre des villes, aussi près que possible des gares de chemin de fer afin d'éviter les contacts avec la population locale et à proximité immédiate des boulevards d'embarquement maritime pour réduire au maximum le trajet du train jusqu'au départ du navire transatlantique.

6 La nature du sol qui va recevoir ces constructions est également choisie en fonction de sa perméabilité, un sol imperméable ayant l'avantage de limiter le passage des miasmes souterrains et de l'humidité. Enfin, la nature des murs de construction est sélectionnée suivant sa dureté. On préconise l'aggloméré de béton de ciment ${ }^{7}$ soutenu par une carcasse de béton armé qui fait office de fondations. Il s'agit d'un procédé de fabrication né sous le Second Empire appelé «système Hennebique» du nom de l'ingénieur qui l'inventa. Son brevet de système constructif armé, déposé en 1892, associé à celui d'Edmond Coignet pour le calcul de la construction deux ans plus tard, révolutionne l'univers architectural. Cette mise en œuvre est caractéristique de l'hôtel Atlantique de Cherbourg, réalisé par l'architecte René Levavasseur ${ }^{8}$ en 1922 pour le compte de trois compagnies maritimes internationales associées (Cunard Line, Star Line et Red Star Line). L'aggloméré de béton règne en maître dans cette construction, depuis les fondations jusqu'aux tuiles de couverture (fig. 2). 


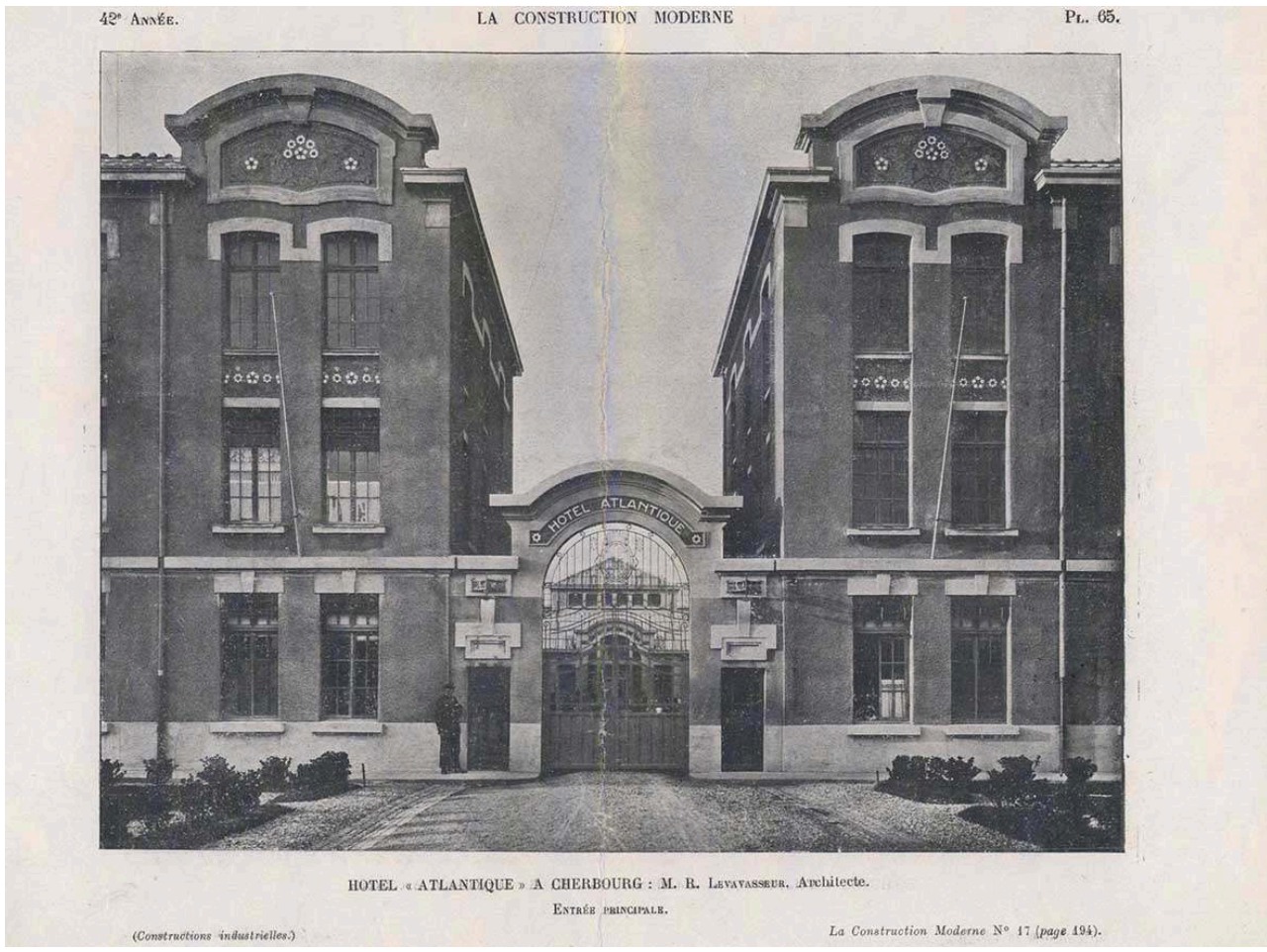

Hôtel Atlantique de Cherbourg. Dans PLANAT, Paul. «L'hôtel Atlantique ». La Construction moderne [Texte imprimé] ; n 17, 23 janvier 1927, p. 194.

7 Toujours pour répondre aux principes hygiénistes, d'autres recommandations sont également faites: elles concernent la mise en place d'un type architectural devant strictement respecter la division entre les zones de désinfection et celles d'hébergement. Par conséquent, la construction de deux bâtiments indépendants, regroupant des zones fonctionnellement dissociées, est organisée. Le premier a une vocation sanitaire, il correspond à une zone attribuée aux migrants arrivants, dits « infectés ». Il regroupe l'accueil (salle d'attente ou de repos, salle à manger), un espace de soins et d'examens médicaux (vestiaires, douches, garde-robe, bureaux du médecin, salon de coiffure), et des réserves (bagagerie, chauffage, salle de désinfection). Il s'agit d'un bâtiment fonctionnel dans lequel tous les services sont ordonnés autour du cabinet médical, centre névralgique à partir duquel rayonnent les divers contrôles sanitaires. Ce bâtiment bénéficie d'une aération zénithale naturelle grâce à l'installation en toiture de lanterneaux facilitant la ventilation et le renouvellement de l'air des ces espaces confinés. Le second espace n'a qu'une fonction hôtelière. Il est structuré en fonction de son usage, l'hébergement des migrants partants dits "désinfectés». Il comprend divers espaces communautaires (réfectoires, cuisines, dortoirs, bibliothèques, buanderie, service de change, magasins d'alimentation, infirmerie et occasionnellement hôpital). Il se caractérise par le recours à une technicité innovante pour les appareils sanitaires tels que les siphons d'évacuation en céramique de grès vernissé dans les pièces d'hygiène corporelle, ainsi que la préconisation du système d'écoulement des eaux par le «tout à l'égout ». Il intègre des pièces pour le personnel de l'établissement ainsi qu'un espace administratif (bureaux et logements) ou des locaux techniques (chaufferie, réserves, remises). L'ensemble du bâtiment hôtelier doit également offrir une disposition fonctionnelle des espaces 
internes, mise en œuvre par la simplicité de l'aménagement intérieur et de son mobilier. L'hôtel Atlantique de Cherbourg est très représentatif de cette caractéristique constructive. D'une superficie totale de $12266 \mathrm{~m}^{2}$, cet établissement concrétise la différenciation structurelle entre deux bâtiments indépendants, l'un, sanitaire (en rouge), pour la désinfection et l'autre, hôtelier (en gris), pour l'hébergement (fig. 3).

Figure 3

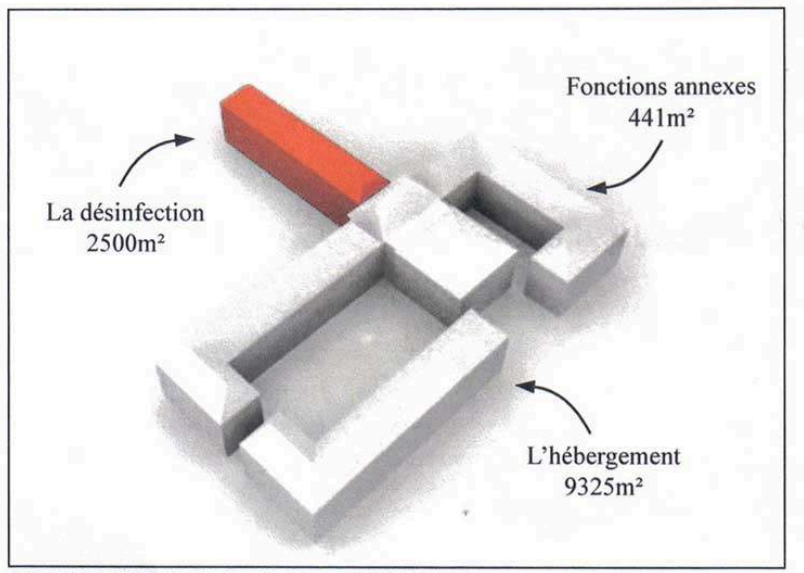

Vue en trois dimentions des surfaces correspondantes aux différents espaces fonctionnels de l'Hôtel Atlantique de Cherbourg

SUPERFICIE TOTALE : $12266 \mathrm{~m}^{2}$

Plan volumétrique général de l'hôtel Atlantique.

Dess. Bosser, Anne, 2012. (c) Anne Bosser.

8 Du point de vue du second œuvre, la prescription architecturale préconise pour les deux édifices, sanitaire et hôtelier, l'utilisation de matériaux hygiénistes dont les performances avaient été mises en lumière dès 1878 grâce aux résultats d'expériences menées par des scientifiques européens. Ils suscitent l'intérêt des architectes qui les recommandent dans le programme des nouveaux établissements à vocation sanitaire. Leur valeur scientifique ayant été reconnue par le corps médical, leur mise en œuvre dans l'aménagement intérieur des espaces collectifs se généralise à travers l'Europe, et les architectes, missionnés par les compagnies maritimes transatlantiques, suivent les recommandations du moment. Sept matériaux destinés aux revêtements de sol et aux parements sont sélectionnés pour leurs caractéristiques physico-hygiénistes: l'asphalte, le granit, la pierre volcanique, le béton, le chêne, le linoléum et le grès cérame. Leur facilité d'entretien, leur durabilité, leur imperméabilité, leur résistance aux impacts, aux chocs et aux produits chimiques (solvants, acides ou huiles), et leur tenue à l'abrasion justifient leur prescription. Pour les finitions des cloisons intérieures, les maîtres d'œuvre optent pour le badigeon au chlorure de chaux ou la peinture à l'huile. Leurs caractéristiques justifient parfaitement le propos du Dr Napias qui corrobore, en se référant aux expériences des scientifiques internationaux, les points de vue des médecins européens sur les qualités exigées par l'hygiène pour tous les revêtements de sols :

«Les murs et les plafonds, dont les angles rentrants sont arrondis, sont couverts de badigeon à la chaux, renouvelé aussi souvent que cela est nécessaire dans l'intérêt de l'hygiène [...] de manière à ne laisser nulle part aucun nid de poussière pouvant servir de réceptacle aux germes volatiles de la virulence. $»^{9}$

D'autres caractéristiques se manifestent dans la prescription architecturale. Elles se rapportent à la mise en œuvre d'innovations techniques comme le chauffage et la 
ventilation, l'alimentation en eau potable, l'éclairage et l'évacuation des eaux et des matières usées. Pour l'aération ambiante, le choix de salles spacieuses, hautes de plafond, bien éclairées et pourvues de larges orifices d'aération s'ouvrant sur des faces opposées de manière à favoriser la ventilation naturelle, est déterminant. La ventilation par propulsion évite les rentrées d'air extérieur. Chaque pièce est munie de fenêtres avec vasistas ou de vitres perforées par lesquelles l'air insufflé peut librement s'échapper. Dans l'hôtel pour migrants d'Amsterdam, le Lloyd Hotel, construit en 1918 par l'architecte Evert Breman pour la compagnie nationale néerlandaise Koninklijke Hollandsche Lloyd, la salle de conversation, par exemple, dispose d'un volume important. Cet espace bénéficie de l'implantation régulière de vasistas à la partie supérieure des fenêtres en périphérie afin de garantir une bonne aération (fig. 4).

Figure 4

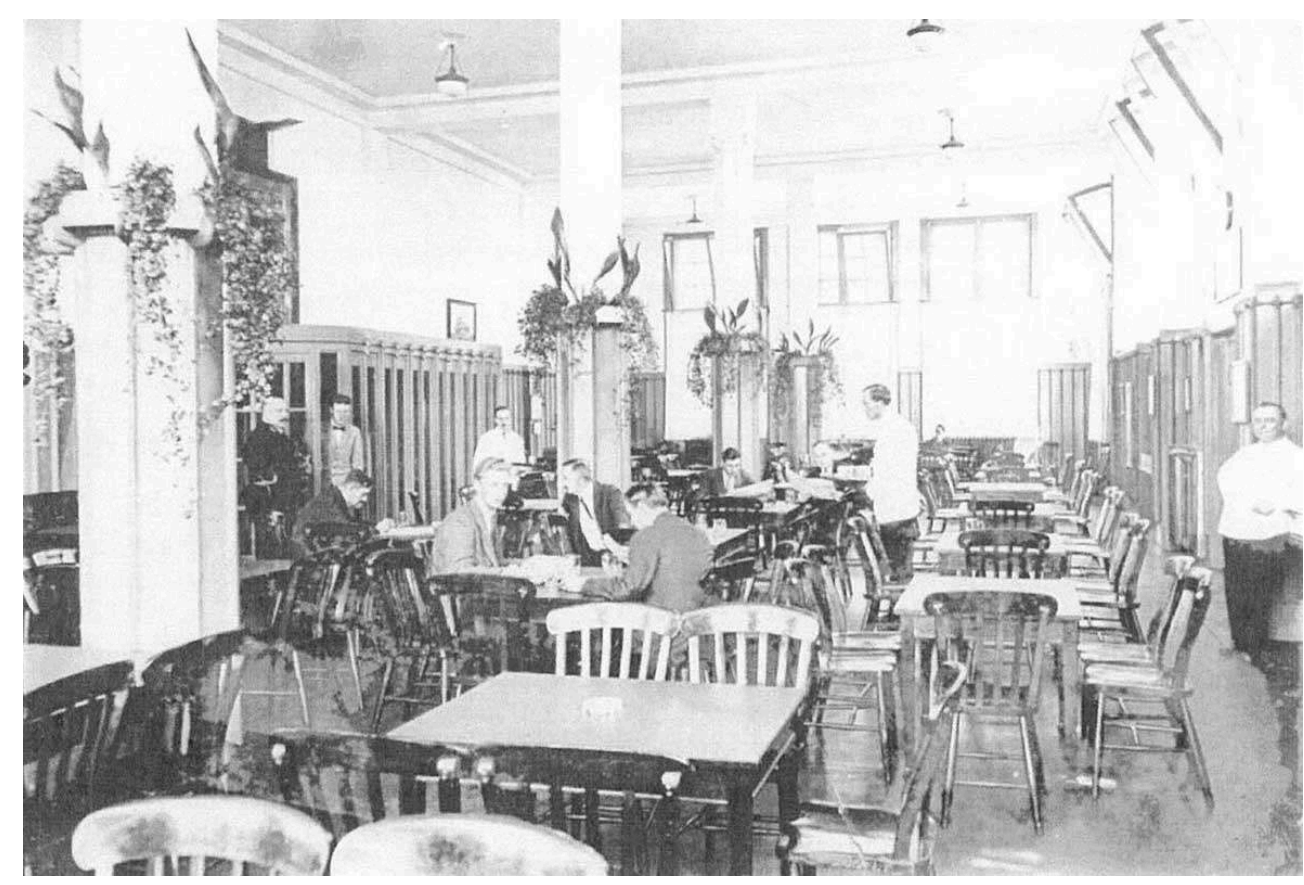

Salle de conversation du Lloyd Hotel d'Amsterdam.

(c) Archives du Musée maritime de Rotterdam, Inventaire F51416, coll. Koninklijke Nedlloyd Groep, 1922.

Pour l'hygiène corporelle, une alimentation en eau chaude à chaque étage des nouveaux bâtiments est recommandée ainsi qu'une installation des équipements sanitaires directement reliés au tout-à-l'égout et au vide sanitaire. Concernant l'assainissement, il est recommandé d'enterrer les conduites d'évacuation des eaux usées afin de protéger des contaminations le sol urbain et les nappes phréatiques et de répondre aux principes de salubrité publique.

11 L'ensemble de ces préconisations permet de faire apparaître un déterminisme spatial cohérent, une conformité hygiéniste que les compagnies maritimes internationales intègrent, à partir du début $\mathrm{du} \mathrm{xx}^{\mathrm{e}}$ siècle, pour la construction de bâtiments spécifiques adaptés au transit de la population. Ces bâtiments prennent en compte simultanément la problématique de santé publique et la montée des préoccupations hygiénistes. Ils révèlent ainsi l'étroite collaboration entre le corps médical et l'architecte prescripteur, 
pour lequel les innovations sanitaires et techniques doivent contribuer à une architecture de qualité.

Dès 1921, à la suite de la promulgation par les États-Unis de diverses lois sur les quotas, cet élan architectural est stoppé et la construction, en Europe, de ces structures d'hébergement temporaire dédiées aux émigrants est enrayée. De nos jours, les établissements de Cherbourg et d'Amsterdam ont acquis un statut patrimonial à la suite de reconversions d'envergure. L'hôtel Atlantique de Cherbourg, restructuré en 1991, accueille les bureaux de la chambre de commerce et d'industrie du Cotentin. Le Lloyd Hotel d'Amsterdam, transformé entre 1999 et 2004 par l'agence d'architecture M.V.R.D.V, est devenu un hôtel de luxe associé au Centre culturel des Pays-Bas. Ces deux témoignages culturels constituent une mémoire architecturale particulière qui se réfère à une période spécifique dans l'histoire de l'émigration européenne.

\section{NOTES}

1. - Article tiré d'une thèse intitulée La spécificité architecturale des lieux de transit portuaire pour migrants d'Europe de l'Ouest entre 1849 et 1934 à partir des cas de Trieste, Amsterdam et Cherbourg. Thèse de doctorat, histoire moderne et contemporaine. Le Havre : université du Havre, 2013.

2. - CARR-SAUNDERS, Alexander Morris. World population, past growth and present, trends. Oxford: The Clarendon Press, 1936, p. 49.

3. - Aujourd'hui Bajoran (Russie), Iłowo-Osada (Pologne), Tcherniakhovsk (Kaliningrad, Russie), Poznan (Pologne), Sovetsk (Kaliningrad, Russie).

4. - Aujourd'hui Bingerbrück (Rhénanie-Palatinat), Myslovitz (Pologne), Racibórz (Pologne).

5. - ANTIN, Mary. From Plotzk to Boston. Boston: W. B. Clarke, 1899, p. 42.

6. - DILLINGHAM, William P., United States Immigration Commission. Reports of the Immigration Commission. Vol. 4: Emigration Conditions in Europe. Washington D.C.: U.S. Government printing Office, 1911, p. 95.

7. - MONNIER, Gérard. L'architecture du XXe siècle. Paris : PUF, coll. « Que sais-je ? ", 2000.

8. - Voir sa notice biographique dans le Dictionnaire des élèves architectes de l'École des beaux-arts : http://www.purl.org/inha/agorha/002/82823 [consulté le 12/10/2016].

9. - NAPIAS, Dr Henri, MARTIN, André-Justin. L'étude et les progrès de l'hygiène en France de 1878 à 1882. Paris : Masson, 1882.

\section{RÉSUMÉS}

À partir de la fin du XIX ${ }^{\mathrm{e}}$ siècle et jusqu'aux lendemains de la Première Guerre mondiale, et plus particulièrement de 1849 à 1924, l'épisode de « la grande émigration européenne » a donné lieu à un exode massif de migrants précaires, en partance pour les Amériques. Ce transit migratoire 
devient, pour les compagnies maritimes transatlantiques internationales, une manne commerciale, et le transport de l'émigrant le plus indigent leur principal bénéfice. Dès lors, elles s'octroient l'exclusivité de la réglementation de l'émigration dans les ports de départ en Europe. Cette nouvelle orientation commerciale leur permet d'envisager la mise en place d'une politique constructive d'hébergement spécifique, tout en régulant l'attente obligatoire pour l'obtention d'une autorisation d'embarquer. Dans la plupart des territoires portuaires de départ sont construits des dispositifs d'accueil et d'hébergement. Leur but est d'accueillir, sélectionner, désinfecter, contrôler, exporter et/ou rapatrier cet abondant fret humain. Ces établissements à vocation sanitaire et sociale évoluent avec la problématique de santé publique et la montée des préoccupations hygiénistes, ce qui met en lumière la collaboration entre l'architecte et le corps médical ainsi que la transformation de la prescription architecturale par le biais des innovations phytosanitaires et techniques. Ces bâtiments de transit se présentent sous la forme de différents types architecturaux spécifiques, qui ne se manifestent nullement comme une architecture de crise ou de l'urgence mais qui répondent précisément à une volonté sanitaire, politique, commerciale et financière de la part de ses nouveaux maîtres d'œuvre.

From the end of the nineteenth century up to the inter-war period and, more generally, from 1849 to 1924 , the episode of the 'great European emigration' saw a massive exodus of poor migrants leaving Europe for the United States. For transatlantic shipping companies, this migratory movement became a major commercial activity and the transport of indigent migrants a leading source of profits. These companies took over the regulation of emigration in the ports of departure in Europe. This new commercial opportunity led the companies to undertake the construction of specific buildings for accommodating the emigrants, whilst regulating the waiting period prior to obtaining an embarkation permit. In most of these port territories, then, special places of reception and accommodation were built. They were designed for the reception of the candidates for emigration, their selection, disinfection, control, export or repatriation. They dealt, in a word, with an abundant supply of human freight. The sanitary and social functions of these places evolved as issues of public health emerged and as hygiene preoccupations came to the fore. The collaboration between architects and the medical professions became essential, and architectural briefs were changed according to phyto-sanitary and technical innovations. These transit buildings present different and specific architectural forms which are by no means an architectural response to crisis or urgency, but which correspond, precisely, with the sanitary, political, commercial and financial objectives of these new commissioning clients.

\section{INDEX}

Mots-clés : bâtiments de transit, émigration, architecture, hygiénisme, Europe

Keywords : transit buildings, emigration, architecture, hygienics, Europe

\section{AUTEUR}

\section{ANNE BOSSER}

Docteur en Histoire moderne et contemporaine, spécialité Histoire de l'architecture, Université du Havre bosser.anne@neuf.fr 